\title{
Schädigungen und Krankheitsbilder Das Surfactant-System der Lunge
}

\author{
W. Kösters, Innere Abteilung, Maria-Hilf-Krankenhaus Bergheim/Erft \\ (Chefarzt: Dr. med. G. Schulte-Herbrüggen)
}

NOTFALLMEDIZIN 2003; 29: 412-415

Das Surfactant-System der Lunge besteht aus einer lipid- und proteinhaltigen Schicht, die die Lungenbläschen (Alveolen) sowie die zuführenden kleinen Bronchiolen auskleidet. Auf verschiedenste Schädigungen reagiert dieses System kaskadenartig in annähernd gleicher Form, bis hin zum ARDS. Somit kommt diesem System auch unter notfallmedizinischen Aspekten eine wichtige Funktion $z u$, deren anatomische, funktionelle und pathophysiologische Kenntnisse zur Erkennung und Behandlung entsprechender Schädigungen und Krankheitsbilder von grundlegender Bedeutung sind.

Die notfallmäßige und klinische Therapie der lokalen oder systemischen Schädigung wird besprochen und anhand der gängigen Verfahren diskutiert. Außerdem kann je nach Schwere des Krankheitsbildes die exogene SurfactantApplikation zur Anwendung kommen.

B eim Surfactant-System der Lunge handelt es sich um die lipid- und proteinhaltige Innenauskleidung (Phospholipidfraktion) der Lungenbläschen (Alveolen) sowie der zuführenden kleinen

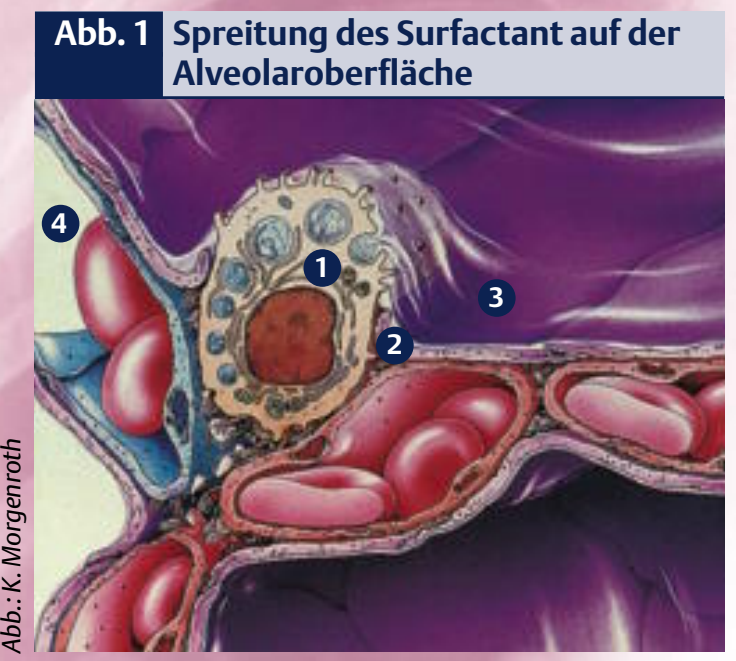

Das von den Pneumozyten II gebildete und auf die Alveolaroberfläche abgegebene Surfactantmaterial spreitet sich als monomolekularer Film über dem Alveolarepithel. Der Surfactant gleicht die Unebenheiten zwischen den Pneumozyten I und II aus. Einzelne Mikrovilli ragen auf der Oberfläche durch den Surfactantfilm in die Alveolarlichtung.

1 Pneumozyt II

2 Pneumozyt I

3 Surfactantfilm

4 Alveolarkapillaren
Bronchiolen. Diese Innenauskleidung hat zahlreiche wichtige, für den Gasaustausch der Lunge unentbehrliche Funktionen. Auf verschiedenste lokale und systemische Schädigungen (thermisch, chemisch, traumatologisch, durch diverse internistische Krankheitsbilder) reagiert dieses System kaskadenartig in annähernd gleicher Form, bis hin zu irreversiblen Veränderungen, sodass der Gasaustausch nachhaltig oder sogar unwiederbringlich behindert werden kann, z.B. ARDS (adult respiratory distress syndrome) im Stadium III und IV.

Somit kommt diesem System auch unter notfallmedizinischen Aspekten eine wichtige Funktion zu, deren anatomische, funktionelle und pathophysiologische Kenntnisse zur Erkennung und Behandlung entsprechender Schädigungen und Krankheitsbilder von grundlegender Bedeutung sind. Die notfallmäßige und klinische Therapie der lokalen oder systemischen Schädigung besteht in der ursächlichen Behandlung der Grunderkrankungen sowie intensivmedizinischer Überwachung und Therapie. Kortikoide können die Stabilität und Neuproduktion des Surfactant-Systems positiv beeinflussen. Als Ultima Ratio kann bei besonderer Schwere des
Krankheitsbildes die exogene über das Bronchialsystem mittels Bronchoskopie durchgeführte Surfactant-Applikation zur Anwendung kommen, deren Nutzen von verschiedenen anästhesiologischen Schwerpunktkliniken unterschiedlich bewertet wird.

\section{Anatomie}

Das Alveolar-System der Lunge umfasst eine Gesamtfläche von zirka $100 \mathrm{~m}^{2}$, die Alveolen werden gleichmäßig von Epithelzellen - überwiegend des Typs Pneumozyten I - bedeckt. Nur 5\% der Epithelfläche werden von Pneumozyten II bedeckt (Abb. 1, Abb. 4). Das gesamte System ist vom Surfactant-Film überzogen, der im Wesentlichen dafür sorgt, dass die Alveolen bei der Exspiration nicht kollabieren und die Wandspannung aufrecht erhalten wird. Während die Pneumozyten I lediglich eine Auskleidungsfunktion besitzen, wird von den Pneumozyten II das zur Auskleidung wesentliche Surfactant gebildet. Neben der Surfactant-Bildung kann sich der Pneumozyt II auch in Pneumozyten I umwandeln, da diese offensichtlich nicht teilungsfähig sind. Die für die Produktion notwendigen Substrate werden aus den umliegenden, zahlreich vorhandenen Kapillaren entnommen (Abb. 1, Abb. 3). Die Surfactant-Membran besteht zu 90\% aus Lipiden, zu 10\% aus Proteinen.

\section{Physiologie}

Die Surfactant-Auskleidung baut eine gewisse Membranspannung auf, ohne die die Alveolen bei jeder Exspiration kollabieren und verkleben würden, sodass sie für den Gasaustausch - von über die Bronchien zugeführten Sauerstoff und das in die Alveolen diffundierte Kohlendioxid - nicht mehr zur Verfügung 
stünden. Somit ist ohne die Surfactant-Auskleidung ein Gasaustausch nicht möglich und die lebenswichtigste Funktion dieses Systems begründet.

Neben der Aufrechterhaltung der Wandspannung hat der Surfactant-Film eine nach außen gerichtete Fließeigenschaft, sodass Verschmutzungen des Alveolar-Bereiches und der kleinsten bronchialen Aufteilungen in Form von Stäuben oder Mikroorganismen durch eine langsame Ausschwemmung beseitigt werden. In den Alveolen und den kleinen Bronchiolen existieren keinerlei Flimmerepithelien mehr, die im sonstigen Bronchialbaum die Reinigungsfunktion durch nach außen gerichteten Flimmerschlag übernehmen. Größere Schmutzpartikel werden vom Surfactant-Film eingeschlossen und den Makrophagen zur Vernichtung angeboten. Ohne die Umschließung durch das Surfactant-System würde die Fresszelle die eingedrungenen Fremdkörper nicht aufnehmen können (Abb. 2, Abb. 4).

Im Rahmen der Infektabwehr übernimmt das Surfactant-System ebenfalls eine wichtige Aufgabe. Viren und Bakterien werden auf die gleiche Art eingeschlossen und den Fresszellen dargeboten, was ohne dieses System nicht ausreichend geschehen würde und virale und bakterielle Pneumonien gehäuft auftreten lassen würde. Darüber hinaus wird die Anlagerung jedweder Fremdkörper an die Bronchial- und Alveolarwand verhindert und somit chronische Entzündungen und die Bildung bösartiger Tumoren erschwert (Abb. 4).

Bei Erreichen der größeren Bronchiolen wird der Surfactant-Film über das Bronchial-System abtransportiert, sodass eine ständige Neuproduktion mit Fließrichtung nach oral erfolgen muss.

Bei Frühgeborenen mit Atemnotsyndrom durch eine unreife Lunge

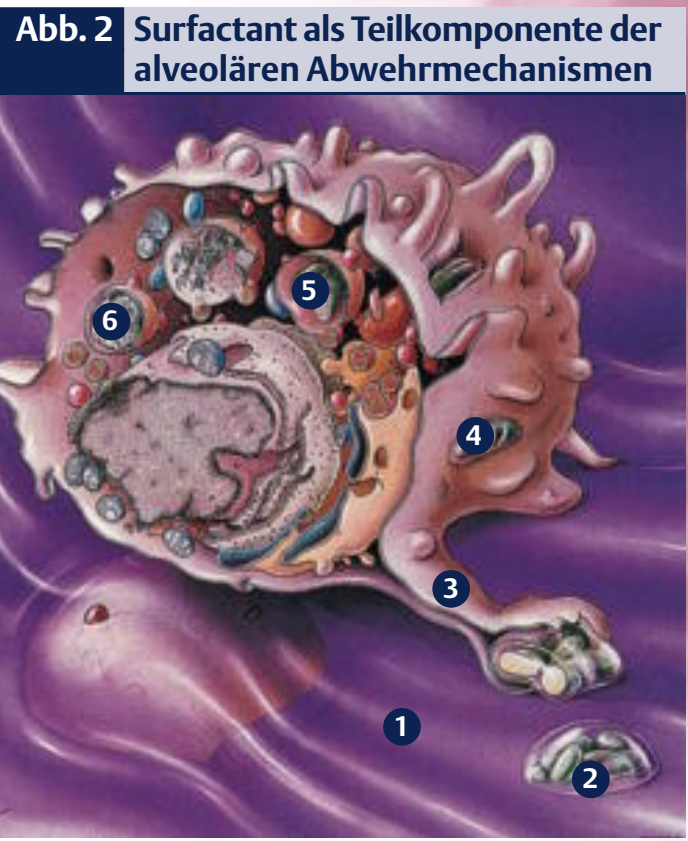

Bakterien werden in der Alveolarlichtung vom Surfactant eingehüllt. Die Makrophagen treten über Zytoplasmaausstülpungen mit den in die Alveolen gelangten Bakterienkomplexe in Kontakt, werden in das Zytoplasma eingeschlossen und in dessen Tiefe verlagert. Hier legen sich primäre Lysosomen an und geben in die Phagosomen lytische Enzyme frei, die den Bakterienabbau ermöglichen.

1 Oberfläche des Alveolarepithels

2 Bakterienkomplex mit Surfactantumhüllung

3 Zytoplasmafortsatz eines Alveolarmakrophagen mit Kontakt zu Bakterienkomplexen

4 Mundförmige Öffnung auf der Oberfläche eines Alveolarmakrophagen

5 Phagosom mit inkorporierten Bakterien und Anlagerung primärer Lysosomen

6 Lytischer Abbau der Bakterien in einem Phagolysom

(Lebensgefahr!) ist das nicht ausgebildete Surfactant-System der entscheidende Faktor für dieses als RDS (neonatal respiratory distress syndrome) bezeichnete Krankheitsbild, wofür die unten aufgeführten exogenen Surfactantprodukte entwickelt und zugelassen wurden.

\section{Pathophysiologie}

Unterschiedlichste Schädigungen der Lunge und des gesamten Organismus führen im Bereich der Alveolen $\mathrm{zu}$ einer gleichartigen Störung des Gleichgewichts zwischen Pneumozyten I und II sowie der Surfactant-Synthese. So führen lokale Schädigungen auf AlveolenEbene - wie lipidlösliche Reizgase oder aspirierte Flüssigkeiten und Infekte, wie virale oder bakterielle Pneumonien - zu einer direkten Schädigung des Surfactant-Filmes der Alveolen. Auch Süß- und Salzwasseraspirationen führen über unterschiedliche Mechanismen zu lokalen Schädigungen des Systems.

Andere Erkrankungen mit vermehrter intestitieller Ödembildung - bei z.B. Sepsis mit vermehrtem Wasseraustritt durch geschädigte Kapillaren, Lungenödem durch Linksherzinsuffizienz aber auch traumatologischen Schädigungen, wie Lungenkontusion, Hitzeschäden bei Inhalationstrauma und Kompression des Lungengewebes durch größere pleurale Flüssigkeits- oder Luftansammlungen bei z.B. Hämato-Pneumothorax (Blut-/Luftansammlung im Pleuraraum) oder ausgedehnten Rechtsherzschwächen mit Pleuraergüssen - führen zu einer gleichartigen Schädigung des Surfactant-Films und dessen unzureichender Neubildung, indem es zur lokalen Schädigung und Oedembildung im Bereich der Alveolen kommt (Abb. 3). Hierdurch wird zum einen der Substratnachschub aus den Blutkapillaren zu den Pneumozyten II gestört, zum anderen kommt es zur schaumartigen Ablösung des Surfactant-Filmes von den Pneumozyten I mit nachfolgend völliger Destruktion des Surfactant-Films mit Ausbildung von Alveolar-Kollaps und Verklebezuständen. Im weiteren Verlauf bilden sich eiweißhaltige hyaline Membranen aus, die den Gasaustausch vollends verhindern, sodass die betroffenen Lungenabschnitte am Gasaustausch nicht mehr teilnehmen können. Je größer diese Abschnitte sind, desto ausgeprägter ist die respiratorische Schädigung und somit die Gefährdung des Patienten.

Diese einmal in Gang gesetzte respiratorische Schädigung führt zu einer reflexartigen Umgehung der 
nicht mehr am Gasaustausch teilnehmenden Lungenabschnitte mit zunehmendem arterio-venösem Shuntvolumen, was zu einer weiteren Reduktion des Gasaustauschs und einer vermehrten Volumen- und Druckbelastung des Herzens führt (von-EulerLiljestrand-Mechanismus). Die vermehrte Volumenbelastung des Herzens kann nun ihrerseits zu einer Linksherzinsuffizienz mit Lungenödem oder einer Rechtsherzinsuffizienz mit Pleuraergüssen führen, die zu einer weiteren Beeinträchtigung der Lungenfunktion führen. So wird aus einer oft lokalen respiratorischen Schädigung eine kardio-zirkulatorische und respiratorische Systemerkrankung.

Andere mögliche Auslöser einer Schädigung des Surfactant-Systems sind Grunderkrankungen mit schwerem Schock („Schocklunge“), Polytraumen auch ohne primäre Lungenschädigung, Intoxikationen, hämorrhagisch nekrotisierende Pankreatitis und extreme Volumenbelastungen nach Massentransfusionen. Das Endbild einer solchen Schädigung ist das ARDS, welches in vier Stadien eingeteilt wird:

- Im Stadium I liegt nur das auslösende Ereignis, noch ohne klinische Symptome vor.

- Das Stadium II weist eine Hyperventilation mit respiratorischer Alkalose, respiratorischer Partialinsuffizienz und ein noch unauffälliges Röntgen-Thoraxbild auf.

- Das Stadium III zeichnet sich zusätzlich durch eine respiratorische Globalinsuffizienz und ein schmetterlingsförmiges interstitielles Lungenödem im RöntgenThoraxbild aus.

- Im Stadium IV entwickelt sich ein zunehmender arterio-venöser Shunt, eine zunehmende Hypoxie, eine erhebliche Schocksymptomatik und ein Multiorganversagen (MOV).

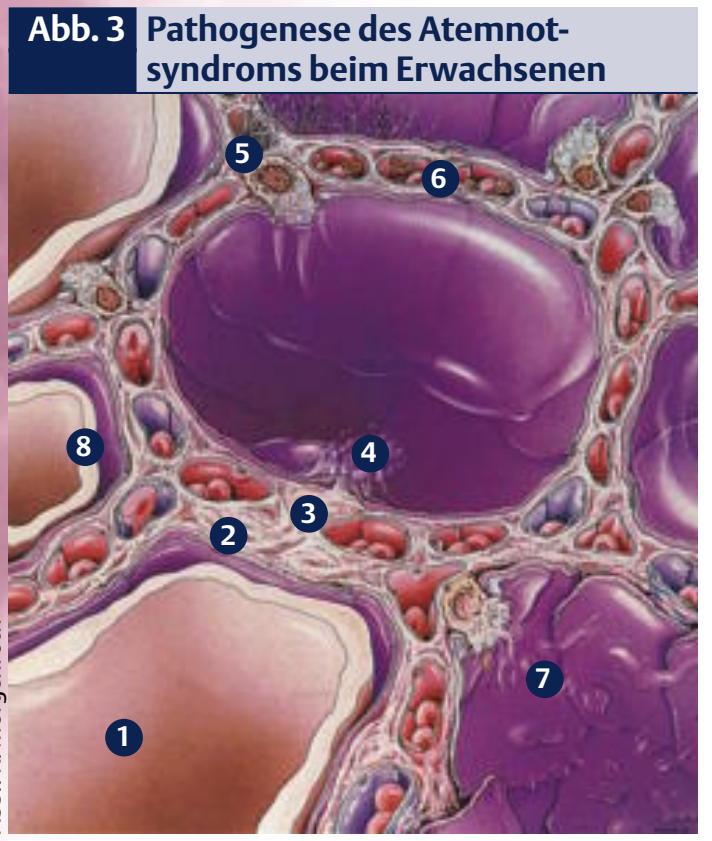

In der Initialphase entsteht ein interstitielles Ödem. Die Ödemflüssigkeit ergießt sich über Spalten im Alveolarepithel in die Alveolarlichtungen und hebt dabei die an der Alveolarwand gespreiteten Surfactantanteile ab. Der Surfactant wandelt sich dabei in eine schaumartige Struktur um. Es entsteht eine Dystelektase und schließlich eine Atelektase der Alveolen. Die fortgeschrittenen Reaktionsstadien sind gekennzeichnet durch Nekrosen des Alveolarepithels, Thrombosen in den Alveolarkapillaren und Bildung hyaliner Membranen.

1 Alveolen

2 Interstitielles Ödem

3 Übertritt des Ödems in die Alveolen

4 Schaumartige Umwandlung des Surfactant

5 Fibrinoide Thrombosen in den Alveolarkapillaren

6 Leukozytenansammlung in den Alveolarkapillaren

7 Nekrosen des Alveolarepithels

8 Hyaline Membranen in den Alveolen

Die Stadien I und II können mit den unten aufgeführten Therapieoptionen meist gut behandelt werden, wobei die Frühzeitigkeit der Behandlung von entscheidender Bedeutung ist. An dieser Stelle muss eine suffiziente präklinische Dokumentation und eine systematisierte Übergabe von Notarzt an Klinikarzt dringend eingefordert werden. Je nach Krankheitsbild ist sogar eine Vorabinformation der aufnehmenden Klinik durch die Rettungsleitstelle oder den Notarzt erforderlich. Die fortgeschrittene Schädigung im Stadium III und IV des AlveolarSystems mit systemischen Auswir- kungen ist trotz aufwendigster Intensivtherapie und differenzierten Beatmungs- und Lagerungstechniken oft nicht mehr behandelbar und führt in vielen Fällen zum Tod des Patienten. Um dies zu verhindern, ist die frühestmögliche Behandlung auch schon präklinisch notwendig.

\section{Therapieoptionen}

Die Notfalltherapie einer solchen Lungenschädigung liegt zum einen in der Behandlung der jeweiligen Grunderkrankung, zum anderen wird durch hochdosierte lokale und systemische Kortikoidgaben versucht, eine Stabilität des Systems zu erreichen und die Pneumozyten II zur erneuten Bildung vom Surfactant zu stimulieren.

Eine frühzeitige auch schon präklinische Intubation und Beatmung mit positiv endexpiratorischem Druck (PEEP) kann eine Verklebung der geschädigten Alveolarabschnitte teilweise verhindern und somit der drohenden Systemerkrankung entgegenwirken. Durch diese Maßnahme wird auch das arterio-venöse Shuntvolumen reduziert.

Intensive Beatmungstechniken - einschließlich der Beatmung in Bauchlage oder im „Schaukelbett“ zur Verhinderung der schwerkraftbedingten Stase der dorsalen und unteren Lungenabschnitte mit weiterer Ausbildung des von-EulerLiljestrand-Mechanismus - sind ein weiterer wichtiger Bestandteil der Therapie. Eine Beatmung mit Stickstoffmonoxid (NO) soll nicht unerwähnt bleiben, wird jedoch in seiner positiven Wirkung unterschiedlich bewertet.

Für Fragen zur Beatmung des ARDS stehen deutschlandweit sechs Zentren mit der Möglichkeit der ECMO (extracorporeal membrane oxygenation) in Aachen, Berlin (Charité, Virchow-Klinikum), Freiburg, Marburg und München-Großhadern 
mit einem 24-Stunden-Service zur Verfügung (Gerlach DMW 2001).

Die rasche Erkennung von Schocksymptomen und deren Bekämpfung mit Pulmonaliskatheter gesteuerter Flüssigkeitsbilanzierung auf niedrig normalem Niveau von 0-3 $\mathrm{cm} \mathrm{H}_{2} \mathrm{O}$, auch unter Einsatz von Katecholaminen, ist ein weiterer wichtiger Therapieansatz. Besonderer Wert ist auf die Notwendigkeit einer ausreichenden gegebenenfalls sogar hochkalorischen möglichst enteralen Ernährung zur Prophylaxe von Unter- und Fehlernährung zu legen. Dies führt zur Verhinderung äußerst schädlicher kataboler Zustände des Organismus mit der Gefahr der Entstehung septischer Krankheitsbilder, der Ausbildung einer Durchwanderungsperitonitis oder der Bildung von Dekubitalgeschwüren, um nur einige negative Auswirkung einer Unter- oder Fehlernährung aufzuzeigen.

Bei fortgeschrittener Erkrankung im Stadium III und IV sind die Therapieoptionen leider nicht mehr wirksam und eine weitere Verschlechterung des Krankheitsbildes mit häufig letalem Ausgang abzusehen. Um dies zu verhindern, ist spätestens in dieser Situation die klinische Gabe von exogenem Surfactant zu diskutieren (Alveofact ${ }^{1}$, Curosurf ${ }^{2}$, Survanta ${ }^{3}$ ). Das exogene Surfactant besteht aus einer Phospholipidfraktion, welche in einem aufwendigen Verfahren aus Rinderlungen gewonnen wird. Neben der Tatsache, dass das exogene Surfactant bisher zur lokalen Applikation einzelner Lungensegmente beim ADRS noch nicht zugelassen ist, schreckt der extrem hohe Preis oft von der Anwendung des exogenen Surfactant ab. Die bisherige Zulassung der oben genannten Präparate beschränkt sich auf das Atemnotsyndrom (RDS) bei unreifer Lunge des Neu- oder Frühgeborenen. Beschreibungen positiver Auswirkungen einer lokalen Applikation bei oben genannten Krankheitsbildern geben Einzelkasuistiken oder persönliche Erfahrungen einzelner Anwender. Schon 1992 berichten $\mathrm{G}$. Buheitel et al. über den erfolgreichen Einsatz von Surfactant bei einem fünf Monate alten Säugling mit schwerer viraler Pneumo- nie. 1999 berichten U. Merz et al. über einen erfolgreichen Einsatz bei einem vierjährigen Jungen nach Verbrennungstrauma und MOV unter Umgehung einer ECMO.

Ob eine frühzeitige, lokalbegrenzte Surfactant-Applikation nicht auf Dauer irreversible Schädigungen verhindern, Menschenleben retten und auch erhebliche Folgekosten reduzieren könnte, erscheint bei entsprechender Betrachtungsweise möglich.

\section{Fazit für die Praxis}

Bei Schädigung des SurfactantFilms durch lokale oder systemische Ursachen erfolgt zunächst die Behandlung der Grunderkrankung. Parallel dazu findet eine frühzeitige präklinische Intubation und PEEPBeatmung statt, sowie intensive klinische physikalische Maßnahmen im Sinne von differenzierten Beatmungs- und Lagerungstechniken, hochdosierte lokale und systemische Kortikoidapplikationen, eine frühestmögliche Schocktherapie und ausgewogene hochkalorische Ernährung. Daneben scheint die lokale Surfactant-Applikation eine letzte Therapieoption darzustellen, die auf Dauer auch einen kostenreduzierenden Effekt haben kann. Eine präklinische Anwendung dieser Substanz kann auf Grund der fehlenden Zulassung beim ARDS und der überwiegend kühlen Lagerung nicht empfohlen werden.

Auf die Notwendigkeit einer suffizienten präklinischen Dokumentation und einer systematisierten Übergabe vom Notarzt an den Klinikarzt darf im Zusammenhang mit den besprochenen Krankheitsbildern hingewiesen werden: Oft präsentieren sich dem Kliniker gut anbehandelte Patienten weniger vital bedroht als dem primär behandelnden Notarzt.

\section{Summary}

The surfactant system in the lungs comprises a lipid- and protein-containing layer that lines the alveoli and the tiny bronchioles. This system reacts in an almost uniform "cascading" fashion in response to a wide range of noxae, and ARDS may finally ensue. The system therefore has an important function - also under emergencymedical aspects - and a knowledge of its anatomical, functional and physiological aspects is essential for recognizing and treating relevant injuries and illnesses.

The present article describes the emergency and clinical treatment of local or systemic injuries, and discusses them in relation to the currently available measures. In addition, depending on the severity of the clinical picture, the application of endogenous surfactant may be indicated.

\section{Anschrift des Verfassers \\ Dr. med. Wolfgang Kösters \\ Facharzt für Innere Medizin/Sportmedizin LNA-Gruppe Erftkreis \\ Innere Abteilung \\ Maria-Hilf-Krankenhaus \\ Klosterstr. 2 \\ 50126 Bergheim/Erft \\ Fax: $02271 / 87215$ \\ ${ }^{1}$ Boeringer Ingelheim Pharma $\mathrm{GmbH} \& \mathrm{Co}$. $\mathrm{KG}$, Ingelheim am Rhein \\ 2 Nycomed Pharma GmbH, Unter- \\ schleißheim \\ ${ }^{3}$ Abbott GmbH \& Co. KG, Wiesbaden}

\section{Abb. 4 Funktionen des Surfactant}

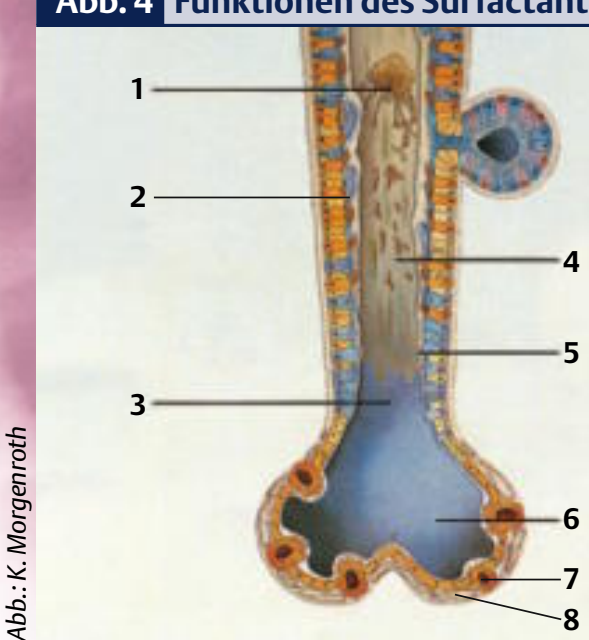

1 Unterstützung der Phagozytose

2 Ablösen von Schleimplaques vom respiratorischen Epithel

3 Abtransport von Fremdpartikeln im flimmerzellenfreien Bereich

4 Schutz des respiratorischen Epithels vor dem Anheften virulenter Bakterien

5 Bildung einer Gleitschicht zwischen Epithel und Sekretstrom

6 Stabilisierung der Alveolen

7 Pneumozyten, Typ II sezernieren Surfactant

8 Pneumozyten, Typ I 